\title{
Hepatic Resection Provides Survival Benefit for Selected Intermediate-Stage (BCLC-B) Hepatocellular Carcinoma Patients
}

\section{Zhang Zhaohui, MD \\ Shen Shunli, PhD \\ Chen Bin, PhD \\ Li Shaoqiang, PhD \\ Hua Yunpeng, PhD \\ Kuang Ming, PhD \\ Liang Lijian, PhD \\ Peng Bao Gang, PhD}

Department of Hepatic Surgery, First Affiliated Hospital, Sun Yat-Sen University, Guangzhou, China

\section{Correspondence: Peng Bao Gang, PhD \\ Department of Hepatic Surgery, \\ First Affiliated Hospital, Sun Yat-Sen \\ University, Guangzhou 510080, China \\ Tel: 86-20-87755766-8214 \\ Fax: 86-20-87755766-8663 \\ E-mail: pengbaogang@medmail.com.cn}

Received January 12, 2018

Accepted February 20, 2018

Published Online February 26, 2018

*Zhang Zhaohui and Shen Shunli contributed equally to this work.

\begin{abstract}
Purpose
The intermediate stage of hepatocellular carcinoma (HCC) (Barcelona Clinic Liver Cancer [BCLC] B) comprises a highly heterogeneous population, and the treatment strategy is still controversial. Because of the heterogeneity, a subclassification of intermediate-stage HCCs was put forward by Bolondi according to the 'beyond Milan and within up-to-7' criteria and Child-Pugh score. In this study, we aim to analyze the prognosis of BCLC-B stage HCC patients who received hepatic resection according to the Bolondi's subclassification.
\end{abstract}

\section{Materials and Methods}

One thousand and one hundred three patients diagnosed with $\mathrm{HCC}$ and treated with hepatic resection were enrolled in our hospital between 2006 and 2012. According to Bolondi's subclassification, the BCLC-B patients were divided into four groups. Recurrence-free survival (RFS) and overall survival (OS) were analyzed.

\section{Results}

According to Bolondi's subclassification, the BCLC-B patients were divided into four groups: B1 $(n=41,18.7 \%)$, B2 ( $n=160,73.1 \%), B 3(n=11,5.0 \%)$, and B4 $(n=7,3.2 \%)$. Significant difference was observed between $B 1$ and other groups (B1 vs. $B 2, p=0.022$; $B 1$ vs. B3, $p$ $<0.001$; B1 vs. $B 4, p<0.001)$, but no difference for B2 vs. $B 4(p=0.542)$ and $B 3$ vs. $B 4$ $(p=0.542)$. In addition, no significant differences were observed between BCLC-A and BCLCB1 group for both RFS ( $p=0.087$ ) and OS ( $p=0.643)$. In multivariate analysis, $B C L C-B$ subclassification was not a risk factor for both OS $(p=0.263)$ and RFS $(p=0.892)$.

\section{Conclusion}

In our study, HCC patients at B1 stage were benefited from hepatic resection and had similar survival to BCLC-A stage patients. Our study provided rationality of hepatic resection for selected BCLC-B stage HCC patients instead of routine transarterial chemoembolization.

\section{Introduction}

Hepatocellular carcinoma (HCC) is the fifth most prevalent neoplasm and the third most frequent cause of cancer mortality in the world [1]. Despite improvements in diagnosis and treatment of surgical techniques and perioperative care, the prognosis of HCC remains far from satisfactory [2].

The Barcelona Clinic Liver Cancer (BCLC) system is widely used for prognosis prediction and treatment strategy
Key words

Hepatocellular carcinoma, Hepatectomy,

Barcelona Clinic Liver Cancer, Bolondi's subclassification selection [3,4]. According to the criteria, hepatic resection and liver transplantation are recommended for early stage tumors (stage 0 and stage $\mathrm{A}$ ), while patients on intermediate-stage are only suitable for palliative treatment, like transarterial chemoembolization (TACE). However, the intermediate stage of HCC (BCLC stage B) comprises a highly heterogeneous population, and the treatment strategy is still controversial [5].

According to the BCLC staging system, the hepatic resection should not be recommended to the patients with BCLC- 
B patients, while retrospective studies have demonstrated that hepatic resection is superior to palliative treatments (TACE) for BCLC-B patients [6,7]. A research group in Italy analyzed the treatment selection and prognosis of $405 \mathrm{HCC}$ cases in the BCLC stage B by the Italian Liver Cancer group [8]. Only $40 \%$ of HCC cases in the BCLC stage B underwent TACE. However, TACE couldn't obviously prolong overall survival (OS) than hepatic resection (median survival, 27 months vs. 37 months). Similarly, Vitale et al. [9] conducted a retrospective study involving 2090 HCC cases in the different BCLC stage patients who were enrolled between 2000 and 2012 by the Italian Liver Cancer group. They revealed that BCLC-B patients may benefit most from hepatic resection than non-surgical treatments. The focus of disputes is the heterogeneity of BCLC-B patients over tumor load, age, liver function, and possible comorbidities.

Because of the heterogeneity, a subclassification of intermediate-stage HCCs was put forward by Bolondi et al. [10] in 2012 according to the 'beyond Milan and within up-to-7' criteria, Child-Pugh score, patient's performance status (tumor-related) and portal vein thrombosis. The novel concept divided the BCLC-B patients into four subgroups (Table 1). Different from the recommendation of classical BCLC staging system, this system recommends liver transplantation or hepatic resection as the first treatment option for B1 patients. It recommends sorafenib or TACE for B2-B4 patients, which is consistent with the classical BCLC staging system. However, the usefulness of the subclassification of BCLC-B is also controversial. Weinmann et al. [11] analyzed 254 BCLC-B patients receiving TACE. The median OS for stage B1-B4 were 28.5, 22.8, 12.3, and 5.9 months, but the logrank test showed no significant survival differences in subclassification. On the contrary, another study conducted by Giannini et al. [12] assessed the prognosis of 269 untreated HCC patients observed in the period 1987-2012. They found the median survival progressively decreased from stage B1 (25 months) through stages B2 (16 months) and B3 (9 months), to stage B4 (5 months, $\mathrm{p}<0.001$ ) and a significantly different survival between contiguous stages. Furthermore, a recent study reported a modified Bolondi's subclassification system (Kinki criteria) [13] combining B2 and B3 group, but no more studies verified the effectiveness of Kinki criteria.
In this study, we aim to analyze the prognosis of BCLC-B stage HCC patients who received hepatic resection according to the Bolondi's subclassification and explore the rationality of hepatic resection for BCLC-B HCC patients.

\section{Materials and Methods}

\section{Study population}

Patients diagnosed with HCC and received hepatectomy were enrolled in our hospital between 2006 and 2012. The final diagnosis of HCC was confirmed by pathological examination of the specimen. All patients were $>18$ years of age, with complete clinical and laboratory data. We collected the clinicopathological variables including sex, age, hepatitis B surface antigen (HBsAg), $\alpha$-fetoprotein (AFP), capsulation, lymphatic metastasis, tumor size. As for platelet count, we used the standard of $150 / \mathrm{mm}^{3}$ as the cutoff value [14,15]. The following criteria were considered to help diagnose HCC : a history of chronic hepatitis, the imaging tests (computed tomography $[\mathrm{CT}]$ or magnetic resonance imaging scan of the abdomen) and the level of AFP. The exclusion criteria were as follows: (1) patients with mixed HCC and cholangiocarcinoma; (2) patients with no follow-up data; and (3) patients receiving TACE or Sorafenib before or after operation.

\section{Subclassification of BCLC-B stage}

Patients were divided into three groups: BCLC-A, BCLC$\mathrm{B}$, and BCLC-C according to the American Association for the Study of the Liver Guidelines (AASLD) [16]. And patients with Child-Pugh A and B liver function, multifocal HCC (two to three tumors $>3 \mathrm{~cm}$ in maximal diameter or more than three tumors regardless of size) and lacking of vascular invasion comprise the intermediate-stage disease (BCLC-B) [10]. Furthermore, a novel classification system proposed by Bolondi divided the BCLC-B patients into four subgroups from B1 to B4 (Table 1).

In terms of the modified Bolondi's subclassification system,

Table 1. Proposed subclassification of BCLC-B patients published

\begin{tabular}{lcccc} 
Subclassification & B1 & B2 & B3 & B4 \\
Child-Pugh points & 5-6-7 & $5-6$ & 7 & $8-9$ \\
Beyond Milan and within Up-to-Seven criteria & In & Out & Out & Out \\
ECOG (tumour-related) PST & 0 & 0 & 0 & $0-1$ \\
Portal vein thrombosis & No & No & No & No \\
\hline
\end{tabular}

BCLC, Barcelona Clinic Liver Cancer; ECOG, Eastern Cooperative Oncology Group; PST, performance status test. 
the subclasses B1, B2, and B3 refer to Child-Pugh scores of 5-7 points and 'in' in terms of the 'up-to-7' criteria; subclass B2 refers to Child-Pugh scores of 5-7 points with 'out' of the 'up-to-7' criteria, and subclass B3 refers to Child-Pugh scores of 8-9 points with either 'in' or 'out' of the 'up-to-7' criteria, respectively.

\section{Surgical procedures}

Hepatic resection, including subsegmentectomy, segmentectomy, bisegmentectomy, trisegmentectomy, right trisectionectomy, right hepatectomy, and left hepatectomy, was performed as proposed by the Committee of the International Hepato-Pancreato-Biliary Association of Brisbane 2000 system [17]. Tumor thrombectomy or combined diaphragmatic resection was performed when necessary.

\section{Follow-up}

Patients were regularly followed up at outpatient clinics.
Patients received a physical examination, liver ultrasound, chest X-ray, and serum AFP test at each follow-up. Abdominal CT scan was performed every 6-12 months or when recurrence was suspected. Recurrence was defined as the emergence of clinical, radiological, and/or pathologic diagnosis of the tumor from a previous origin locally or distantly. Once recurrence was confirmed, salvage treatments, including further surgery, percutaneous ablation, or transcatheter arterial chemoembolization, were selected as needed.

\section{Statistical analysis}

Recurrence-free survival (RFS) was calculated from the date of surgery to the date of recurrence, and OS from the date of surgery to the date of HCC-associated death. Survival curves among groups were calculated by the Kaplan-Meier method. Univariate analysis of prognostic variables was performed by log-rank test and multivariate analysis by Cox proportional-hazards model. A p-values of $<0.05$ was considered to be statistically significant. All statistical analyses

Table 2. Main clinical characteristics of patients

\begin{tabular}{|c|c|c|c|c|c|c|c|}
\hline \multirow[b]{2}{*}{ Variable } & \multirow{2}{*}{$\begin{array}{c}\text { All } \\
(n=1,103)\end{array}$} & \multirow{2}{*}{$\begin{array}{c}\text { BCLC-A } \\
(n=530)\end{array}$} & \multicolumn{4}{|c|}{ BCLC-B $(n=219)$} & \multirow{2}{*}{$\begin{array}{l}\text { BCLC-C } \\
(n=354)\end{array}$} \\
\hline & & & $\begin{array}{c}\text { BCLC-B1 } \\
(n=41)\end{array}$ & $\begin{array}{c}\text { BCLC-B2 } \\
(n=160)\end{array}$ & $\begin{array}{c}\text { BCLC-B3 } \\
(\mathbf{n}=\mathbf{1 1})\end{array}$ & $\begin{array}{c}\text { BCLC-B4 } \\
(n=7)\end{array}$ & \\
\hline \multicolumn{8}{|l|}{ Sex } \\
\hline Male & 970 & 444 & 39 & 148 & 11 & 5 & 323 \\
\hline Female & 133 & 86 & 2 & 12 & 0 & 2 & 31 \\
\hline \multicolumn{8}{|l|}{ Age (yr) } \\
\hline$<50$ & 521 & 251 & 17 & 75 & 2 & 2 & 174 \\
\hline$\geq 50$ & 582 & 279 & 24 & 85 & 9 & 5 & 180 \\
\hline \multicolumn{8}{|l|}{ PLT $\left(\times 10^{9} / \mathrm{L}\right)$} \\
\hline$<150$ & 299 & 149 & 15 & 33 & 1 & 2 & 99 \\
\hline$\geq 150$ & 804 & 381 & 26 & 127 & 10 & 5 & 255 \\
\hline \multicolumn{8}{|l|}{ HBsAg } \\
\hline Negative & 157 & 89 & 3 & 19 & 1 & 1 & 44 \\
\hline Positive & 946 & 441 & 38 & 141 & 10 & 6 & 310 \\
\hline \multicolumn{8}{|l|}{$\operatorname{AFP}(n g / m L)$} \\
\hline$<400$ & 543 & 293 & 23 & 77 & 4 & 3 & 143 \\
\hline$\geq 400$ & 560 & 237 & 18 & 83 & 7 & 4 & 211 \\
\hline \multicolumn{8}{|l|}{ Capsulation } \\
\hline Capsulated & 812 & 421 & 36 & 131 & 9 & 7 & 208 \\
\hline Noncapsulated & 291 & 109 & 5 & 29 & 2 & 0 & 146 \\
\hline \multicolumn{8}{|c|}{ Lymphatic metastasis } \\
\hline Absent & 1,030 & 524 & 39 & 150 & 9 & 7 & 301 \\
\hline Present & 73 & 6 & 2 & 10 & 2 & 0 & 53 \\
\hline \multicolumn{8}{|l|}{ Tumor size (cm) } \\
\hline$<5$ & 385 & 249 & 41 & 19 & 1 & 4 & 71 \\
\hline$\geq 5$ & 718 & 281 & 0 & 141 & 10 & 3 & 283 \\
\hline
\end{tabular}

BCLC, Barcelona Clinic Liver Cancer; PLT, platelet; HBsAg, hepatitis B surface antigen; AFP, $\alpha$-fetoprotein. 
A
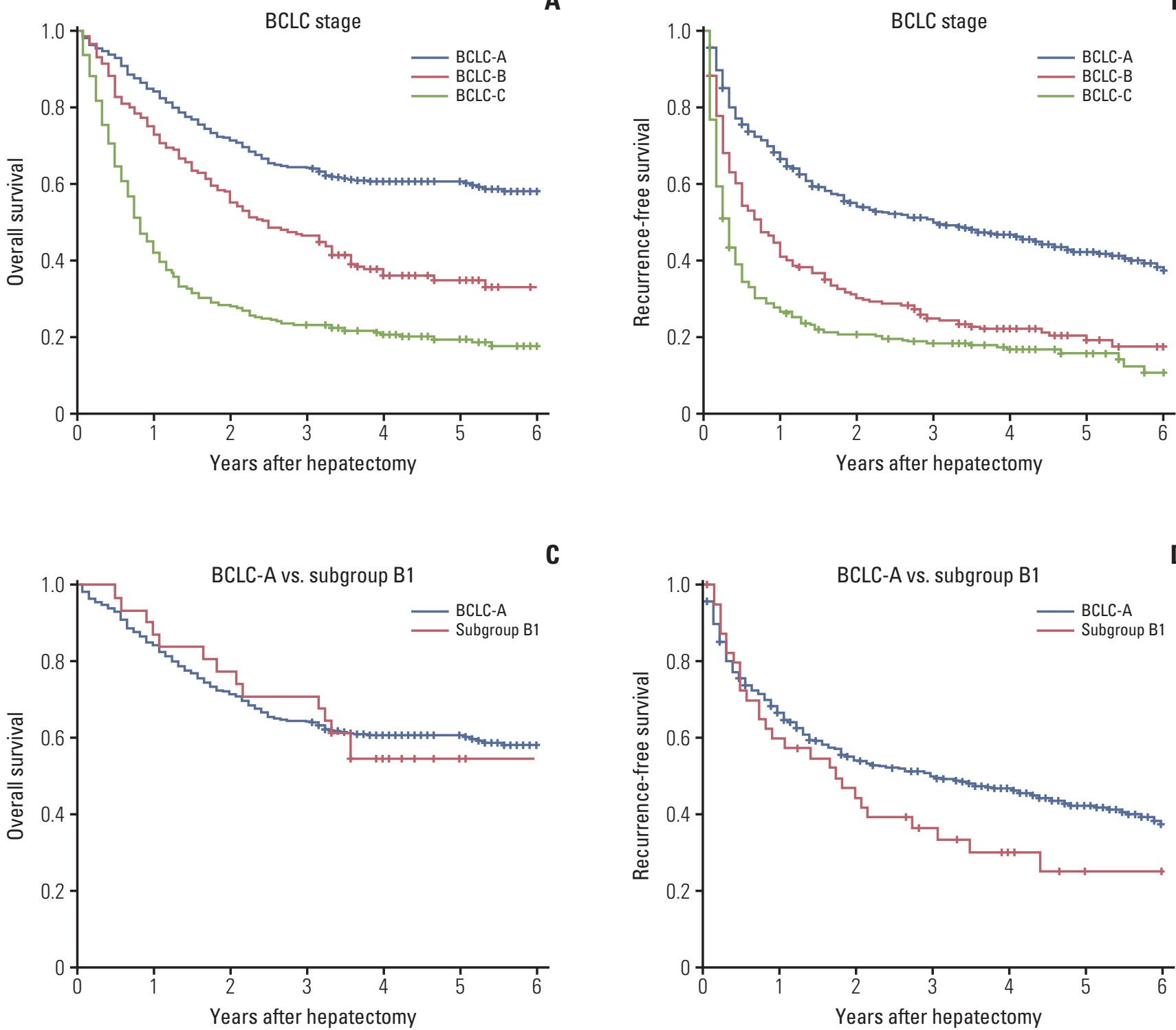

Fig. 1. (A) Overall survival according to the Barcelona Clinic Liver Cancer (BCLC) staging system. (B) Recurrence-free survival according to the BCLC staging system. (C) Overall survival of BCLC-A and BCLC-B1. (D) Recurrence-free survival of BCLC-A and BCLC-B1.

were performed using package of rms in $\mathrm{R}$ ver. 2.14.1 (http://www.r-project.org/).

\section{Ethical statement}

Informed consent was obtained, and procedures were carried out with prior approval of the Ethics Committee of the First Affiliated Hospital of Sun Yat-sen University (Guangzhou, China).

\section{Results}

\section{Patients}

A total of 1,103 patients were eligible for the study. The median age at diagnosis was 50 years (range, 21 to 79 years), and there were 970 men and 133 women. HBsAg was positive in 946 patients $(85.7 \%)$. Increased AFP ( $\geq 400 \mathrm{ng} / \mathrm{mL}$ ) was found in 560 cases $(50.8 \%)$, and 299 patients $(27.1 \%)$ had 

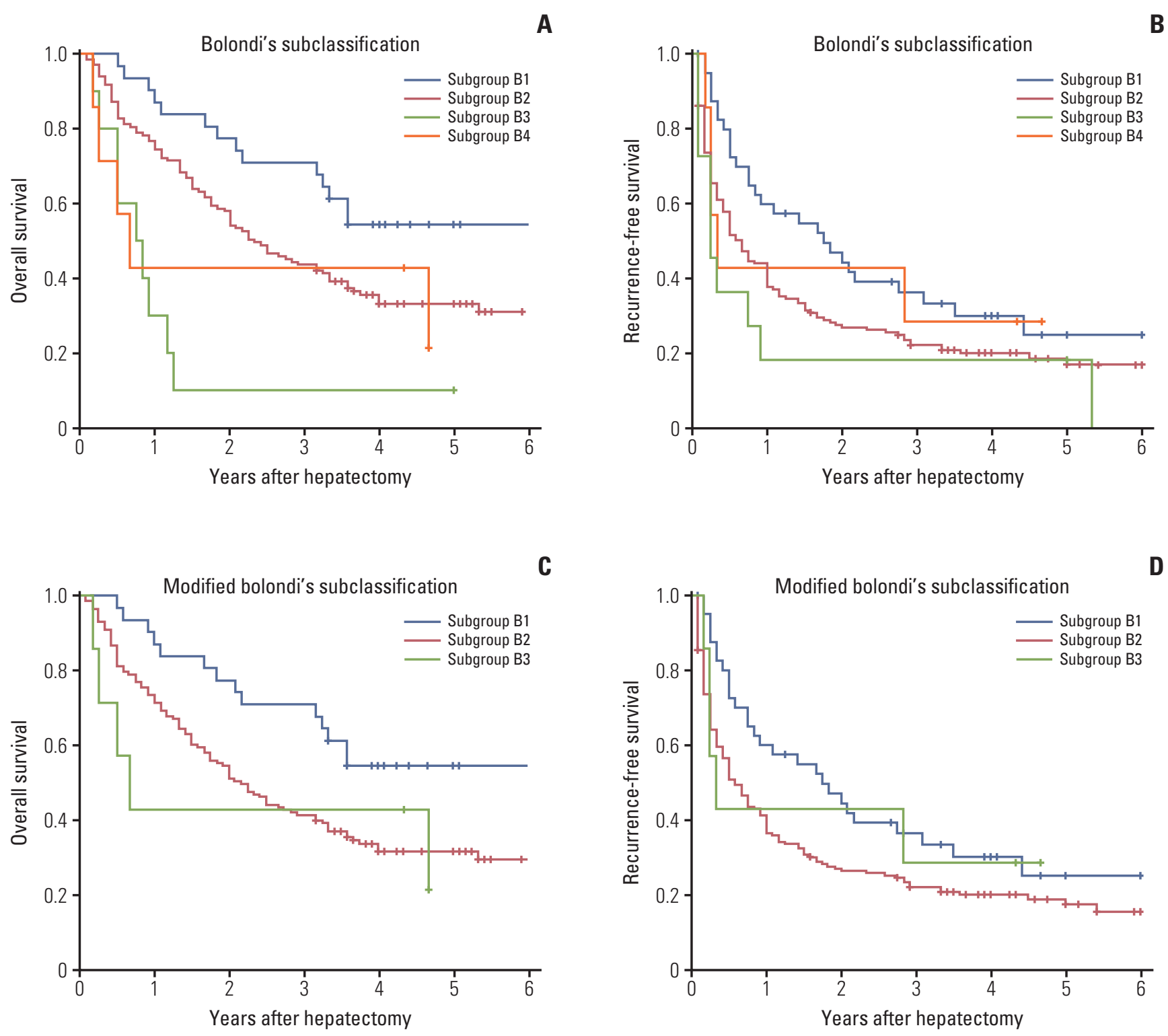

Fig. 2. (A) Overall survival according to the Bolondi's classification. (B) Recurrence-free survival according to the Bolondi's classification. (C) Overall survival according to the modified Bolondi's classification (Kinki criteria). (D) Recurrence-free survival according to the modified Bolondi's classification (Kinki criteria).

a low platelet count $\left(<150 \times 10^{9} / \mathrm{L}\right)$. And 718 patients $(65.1 \%)$ had tumors larger than $5 \mathrm{~cm}$. As to lymphatic metastasis, lymphatic metastasis was found in 73 patients $(6.6 \%)$. Details of features are shown in Table 2. The follow-up time ranged from 4 to 131 months. Seventeen point six percent patients were lost to follow-up.

\section{Survival according to BCLC-stage}

The median RFS and OS were 12 months and 30 months. According to the BCLC stage, we classified the HCC patients into three stages: BCLC-A ( $\mathrm{n}=530,48.1 \%), \mathrm{BCLC}-\mathrm{B}(\mathrm{n}=219$, $19.9 \%)$, and BCLC-C $(n=354,32.1 \%)$. The median survival was 92 months in the BCLC-A, 30 months in BCLC-B, and 10 months in BCLC-C, respectively. Furthermore, the median RFS was 36 months in the BCLC-A, 9 months in BCLC-B, and 4 months in BCLC-C, respectively. A significant difference 
Table 3. Multivariable regression results for overall survival and recurrence-free survival

\begin{tabular}{lccccc}
\multirow{2}{*}{ Characteristic } & \multicolumn{2}{c}{ Overall survival } & & \multicolumn{2}{c}{ Recurrence-free survival } \\
\cline { 2 - 3 } \cline { 5 - 6 } Sex & HR $(95 \% \mathrm{CI})$ & p-value & & HR (95\% CI) & p-value \\
Age & $0.56(0.24-1.32)$ & 0.184 & & $0.36(0.15-0.82)$ & 0.015 \\
PLT & $0.95(0.62-1.45)$ & 0.799 & & $0.98(0.68-1.42)$ & 0.915 \\
HBsAg & $0.77(0.47-1.24)$ & 0.288 & & $1.27(0.82-1.97)$ & 0.289 \\
AFP & $1.02(0.53-1.93)$ & 0.960 & & $1.09(0.63-1.89)$ & 0.764 \\
Capsulation & $1.37(0.88-2.11)$ & 0.160 & & $1.44(0.99-2.08)$ & 0.052 \\
Bolondi's subclassification & $1.51(0.90-2.55)$ & 0.120 & & $1.11(0.70-1.77)$ & 0.643 \\
Lymphatic metastasis & $1.31(0.82-2.10)$ & 0.263 & & $0.97(0.65-1.46)$ & 0.892 \\
Tumor size & $2.01(0.81-4.98)$ & 0.131 & & $1.49(0.70-3.20)$ & 0.299 \\
\hline
\end{tabular}

$\mathrm{HR}$, hazard ratio; CI, confidence interval; PLT, platelet; HBsAg, hepatitis B surface antigen; AFP, $\alpha$-fetoprotein.

was observed between BCLC stage by log-rank test $(\mathrm{p}<0.05)$ (Fig. 1A and B).

\section{Bolondi's subclassification}

According to Bolondi's subclassification, the BCLC-B patients were divided into four groups: $\mathrm{B} 1(\mathrm{n}=41,18.7 \%)$, B2 $(\mathrm{n}=160,73.1 \%), \mathrm{B} 3(\mathrm{n}=11,5.0 \%)$, and B4 $(\mathrm{n}=7,3.2 \%)$. Most of the patients $(\mathrm{n}=201,91.8 \%)$ were $\mathrm{B} 1$ group or $\mathrm{B} 2$ group. The median survival was 75 months, 28 months, 9.5 months, and 8 months for B1-B4 group. Results from the log-rank test showed that obvious difference was observed between $\mathrm{B} 1$ group and other groups (B1 vs. B2, $\mathrm{p}=0.022 ; \mathrm{B} 1$ vs. B3, $p<0.001$; B1 vs. B4, $p<0.001)$, but no difference for B2 vs. $\mathrm{B} 4(\mathrm{p}=0.542), B 3$ vs. $\mathrm{B} 4(\mathrm{p}=0.542)$, and B2 vs. B3 ( $\mathrm{p}=0.645)$ (Fig. 2A).

In addition, the median RFS was 21 months, 8 months, 3 months and 4 months for B1-B4 groups. And results from the log-rank test showed significant survival differences for $B 1$ vs. $B 2$ ( $p=0.041$ ) and B1 vs. $B 3$ ( $p=0.024)$, but no difference for the B2 vs. B3 ( $p=0.274)$ and B3 vs. B4 ( $p=0.483$ ) (Fig. 2B).

In multivariate analysis, Bolondi's subclassification had no significant effect for RFS ( $p=0.892)$ and OS $(p=0.263)$. Tumor size was the only independent risk predictor of OS (hazard ratio [HR], 2.74; 95\% confidence interval [95\% CI], 1.58 to 4.74; $\mathrm{p}<0.001)$ for BCLC-B patients. In addition, tumor size (HR, 2.31; 95\% CI, 1.42 to 3.74; $\mathrm{p}=0.001)$ and sex (HR, 0.36; $95 \% \mathrm{CI}, 0.15$ to 0.82 ) were independent risk predictors $\mathrm{p}=0.015$ ) for RFS (Table 3 ).

\section{Modified Bolondi's subclassification system (Kinki cri- teria)}

The median OS for modified subclassification is 58 months, 14 months and 9 months, and the median RFS is 29 months,
5 months and 3 months. Although obvious difference was observed between $\mathrm{B} 1$ and other groups for OS (B1 vs. B2, $\mathrm{p}<0.001$; B1 vs. B3, $\mathrm{p}<0.001$ ) and RFS (B1 vs. B2, $\mathrm{p}=0.478$; $B 1$ vs. B3, $p<0.001$ ), there was still no obvious difference for RFS ( $\mathrm{p}=0.722)$ and OS ( $\mathrm{p}=0.337)$ between B2 and B3 according to Kinki criteria (Fig. 2C and D).

\section{BCLC-A stage vs. BCLC-B1 group}

Subsequently, the 1-, 3-, and 5-year OS rate was 88,71 , and $68 \%$ for BCLC-A stage and 92\%, 78\%, and $66 \%$ for BCLC-B1 subclassification. Correspondingly, the 1-, 3-, and 5-year recurrence rate was $70 \%, 56 \%$, and $48 \%$ for BCLC-A stage and $59 \%, 40 \%$, and $32 \%$ for BCLC-B1 subclassification. The log-rank test revealed that no significant differences were observed between BCLC-A and BCLC-B1 group for both RFS $(\mathrm{p}=0.087)$ and $\mathrm{OS}(\mathrm{p}=0.643)$ (Fig. $1 \mathrm{C}$ and $\mathrm{D})$.

\section{Discussion}

The BCLC staging system, endorsed by European Association for the Study of Liver (EASL) and AASLD, provides prognostic score and guidance for therapeutic decisions for HCC patients based on tumor staging criteria, liver function and health status $[18,19]$. Intermediate stage HCC (BCLC-B) patients are a very heterogeneous population in terms of tumor size, tumor number and liver function [13]. On account of the heterogeneity, increasing researchers have begun to create a novel subclassification system to improve the staging-treatment association. In 2012, Bolondi et al. [10] proposed an innovative classification system. Thereafter, many studies had been carried out to prove the effectiveness 
of Bolondi's subclassification. A recent study designed by Ciria et al. [20], involving 80 BCLC-B patients received TACE and hepatic resection between 2007 and 2012, revealed that overall survival was $40 \%$ with a median follow-up of 29.5 months (0.07-96.9) and 5-year survival rates were $62.9 \%$, $28.1 \%$, and $15.4 \%$, respectively ( $\mathrm{p}=0.004)$ for $\mathrm{B} 1, \mathrm{~B} 2$, and B3-4 stages.

In our study, we found the overall survival trends were remarkable in B1 stages, but no difference for the test B2 vs. $\mathrm{B} 3$ and B3 vs. B4. In addition, Bolondi's subclassification had no significant effect for RFS ( $p=0.892)$ and OS $(p=0.263)$ in multivariate analysis. This illustrates that Bolondi's subclassification cannot totally distinguish the BCLC-B patients who received hepatic resection, especially the subclass B2 to B4. For this reason, a research from Kinki University School of Medicine in Japan put forward a novel classification system by modifying the Bolondi's subclassification [13]. In this modified Bolondi's subclassification system (Kinki criteria), the biggest change is putting B2 and B3 group together. Then patients with intermediate-stage HCC are classified into 3 groups. Further analysis of validity of the Kinki criteria by Arizumi et al. [21] with 425 HCC patients who underwent TACE, and the result revealed that the median overall survival was 3.9 years in the BCLC subclass B1 group, 2.5 years in the B2 group, and 1.1 years in the B3 group $(p<0.001)$, and survival curves were stratified with significant differences. In our study, there was no obvious difference for RFS $(\mathrm{p}=0.722)$ and $\mathrm{OS}(\mathrm{p}=0.337)$ between B2 and B3 according to Kinki criteria (Fig. 2C and D). Therefore, both of the two subclassifications may be not suitable to distinguish BCLC$\mathrm{B} 2-\mathrm{B} 4$ patients who received hepatic resection. And these findings stress once again that BCLC stage B is a heterogeneous category.

Although TACE was considered as the main treatment for those patients, it is not always the case in the clinical work because of the heterogeneity. Several groups proposed that therapeutic approach is different according to subclassification. As it is recently stated in a study from the Italian Liver Cancer Group in which TACE for BCLC-B was analyzed on 405 patients, "TACE cannot be considered the best approach for BCLC stage B patients who represent a heterogeneous population and are often suitable for more aggressive therapies, which lead to a better survival" [8]. Similarly, Zhong et al. [6] reported a wide single center experience with similar results. In fact, with deepened understanding of liver cirrhotic and improved operative techniques, hepatic resection may be a much more feasible and safe choice for some BCLC$B$ patients. In our study, we found that the BCLC-B1 patients with hepatic resection had a better prognosis than the B2-B4 patients and no significant difference in RFS and OS between the BCLC-A and the subclass B1 group. Although many studies believed the only TACE is indicated for intermedi- ate-stage HCC [22,23], a recent randomized controlled trial (RCT) conducted by Yin et al. [24], including 173 patients with multinodular HCC, revealed that there were no significant differences in the 30-day and in the 90-day mortality between the hepatic resection and TACE groups for BCLC$B$ patients. However, the hepatic resection group had significantly better overall survive than the TACE group. In addition, a meta-analysis including 50 retrieved papers shows a statistically significantly higher overall survival in hepatic resection group than in TACE group in HCC within the BCLC stage B alone (HR, 0.48; 95\% CI, 0.25 to 0.90) [25]. Furthermore, in the research of Ciria et al. [20], no significant difference were found in the HCC patients with BCLC-B1 who underwent live resection with those who received TACE treatment for 5-year survival rate $(63.2 \%$ vs. $62.5 \%)$. Therefore, in our opinion, Bolondi's subclassification may be used to the selection of part of BCLC-B patients who may benefit from hepatic resection. On the other hand, as for patients in the subclass B2-B4, TACE may be a more appropriate treatment, which could reduce the risk of liver failure.

Some limitations have been found in our study. Firstly, the main limitation is the retrospective nature, which may result in selection bias. We have been carrying on a prospective multicenter RCT comparing hepatic resection versus TACE+ radiofrequency ablation for BCLC-B stage HCC patients (RCT: NCT02616926). Secondly, owing to the severe liver function injury and huge tumor burden, there are only six and five patients who received hepatic resection for subclass B3 and B4 and 18.6\% patients were lost to follow up in BCLCB stage HCC patients, which may cause a statistical discrepancy. This is why the median survival of B4 group longer than the B3 group in our study.

In our study, we stress once again that BCLC stage B is a heterogeneous category and the overall survival trends were remarkable in B1 stages according to Bolondi's subclassification for HCC patients with hepatic resection. In addition, no significant difference in RFS and OS between the BCLC-A and the B1 stages were found, suggesting that some BCLC$B$ patients may benefit from hepatic resection. Therefore, our study provided rationality of hepatic resection for selected BCLC-B stage HCC patients instead of routine TACE.

\section{Conflicts of Interest}

Conflict of interest relevant to this article was not reported.

\section{Acknowledgments}

This work was supported by Sun Yat-Sen University Clinical Research 5010 Program (Grant number: 2015012). 


\section{References}

1. Ferlay J, Shin HR, Bray F, Forman D, Mathers C, Parkin DM. Estimates of worldwide burden of cancer in 2008: GLOBOCAN 2008. Int J Cancer. 2010;127:2893-917.

2. Pang Q, Qu K, Zhang JY, Song SD, Liu SS, Tai MH, et al. The prognostic value of platelet count in patients with hepatocellular carcinoma: a systematic review and meta-analysis. Medicine (Baltimore). 2015;94:e1431.

3. Forner A, Llovet JM, Bruix J. Hepatocellular carcinoma. Lancet. 2012;379:1245-55.

4. Llovet JM, Di Bisceglie AM, Bruix J, Kramer BS, Lencioni R, Zhu AX, et al. Design and endpoints of clinical trials in hepatocellular carcinoma. J Natl Cancer Inst. 2008;100:698-711.

5. Chang WT, Kao WY, Chau GY, Su CW, Lei HJ, Wu JC, et al. Hepatic resection can provide long-term survival of patients with non-early-stage hepatocellular carcinoma: extending the indication for resection? Surgery. 2012;152:809-20.

6. Zhong JH, Ke Y, Gong WF, Xiang BD, Ma L, Ye XP, et al. Hepatic resection associated with good survival for selected patients with intermediate and advanced-stage hepatocellular carcinoma. Ann Surg. 2014;260:329-40.

7. Choi SH, Choi GH, Kim SU, Park JY, Joo DJ, Ju MK, et al. Role of surgical resection for multiple hepatocellular carcinomas. World J Gastroenterol. 2013;19:366-74.

8. Farinati F, Vanin V, Giacomin A, Pozzan C, Cillo U, Vitale A, et al. BCLC stage $B$ hepatocellular carcinoma and transcatheter arterial chemoembolization: a 20-year survey by the Italian Liver Cancer group. Liver Int. 2015;35:223-31.

9. Vitale A, Burra P, Frigo AC, Trevisani F, Farinati F, Spolverato $G$, et al. Survival benefit of liver resection for patients with hepatocellular carcinoma across different Barcelona Clinic Liver Cancer stages: a multicentre study. J Hepatol. 2015;62: 617-24.

10. Bolondi L, Burroughs A, Dufour JF, Galle PR, Mazzaferro V, Piscaglia F, et al. Heterogeneity of patients with intermediate (BCLC-B) hepatocellular carcinoma: proposal for a subclassification to facilitate treatment decisions. Semin Liver Dis. 2012;32:348-59.

11. Weinmann A, Koch S, Sprinzl M, Kloeckner R, SchulzeBergkamen H, Duber C, et al. Survival analysis of proposed BCLC-B subgroups in hepatocellular carcinoma patients. Liver Int. 2015;35:591-600.

12. Giannini EG, Moscatelli A, Pellegatta G, Vitale A, Farinati F, Ciccarese F, et al. Application of the intermediate-stage subclassification to patients with untreated hepatocellular carcinoma. Am J Gastroenterol. 2016;111:70-7.

13. Kudo M, Arizumi T, Ueshima K, Sakurai T, Kitano M, Nishida N. Subclassification of BCLC B stage hepatocellular carcinoma and treatment strategies: proposal of modified Bolondi's subclassification (Kinki Criteria). Dig Dis. 2015;33:751-8.

14. Maithel SK, Kneuertz PJ, Kooby DA, Scoggins CR, Weber SM, Martin RC 2nd, et al. Importance of low preoperative platelet count in selecting patients for resection of hepatocellular carcinoma: a multi-institutional analysis. J Am Coll Surg. 2011;212:638-48.

15. Lim C, Compagnon P, Sebagh M, Salloum C, Calderaro J, Luciani A, et al. Hepatectomy for hepatocellular carcinoma larger than $10 \mathrm{~cm}$ : preoperative risk stratification to prevent futile surgery. HPB (Oxford). 2015;17:611-23.

16. Bruix J, Sherman M; American Association for the Study of Liver Diseases. Management of hepatocellular carcinoma: an update. Hepatology. 2011;53:1020-2.

17. Sutherland F, Harris J. Claude Couinaud: a passion for the liver. Arch Surg. 2002;137:1305-10.

18. Dhir M, Melin AA, Douaiher J, Lin C, Zhen WK, Hussain SM, et al. A review and update of treatment options and controversies in the management of hepatocellular carcinoma. Ann Surg. 2016;263:1112-25.

19. EASL-EORTC clinical practice guidelines: management of hepatocellular carcinoma. J Hepatol. 2012;56:908-43.

20. Ciria R, Lopez-Cillero P, Gallardo AB, Cabrera J, Pleguezuelo $\mathrm{M}$, Ayllon MD, et al. Optimizing the management of patients with BCLC stage-B hepatocellular carcinoma: modern surgical resection as a feasible alternative to transarterial chemoemolization. Eur J Surg Oncol. 2015;41:1153-61.

21. Arizumi T, Ueshima K, Iwanishi M, Minami T, Chishina $H$, Kono $\mathrm{M}$, et al. Validation of a modified substaging system (Kinki Criteria) for patients with intermediate-stage hepatocellular carcinoma. Oncology. 2015;89 Suppl 2:47-52.

22. Adhoute X, Penaranda G, Raoul JL, Bourliere M. Hepatocellular carcinoma scoring and staging systems. Do we need new tools? J Hepatol. 2016;64:1449-50.

23. Dufour JF, Bargellini I, De Maria N, De Simone P, Goulis I, Marinho RT. Intermediate hepatocellular carcinoma: current treatments and future perspectives. Ann Oncol. 2013;24 Suppl 2:ii24-9.

24. Yin L, Li H, Li AJ, Lau WY, Pan ZY, Lai EC, et al. Partial hepatectomy vs. transcatheter arterial chemoembolization for resectable multiple hepatocellular carcinoma beyond Milan Criteria: a RCT. J Hepatol. 2014;61:82-8.

25. Qi X, Wang D, Su C, Li H, Guo X. Hepatic resection versus transarterial chemoembolization for the initial treatment of hepatocellular carcinoma: a systematic review and metaanalysis. Oncotarget. 2015;6:18715-33. 